\title{
Technik im familialen Alltag ${ }^{1}$
}

\section{Zur Analyse familienspezifischer Aneignungsmuster}

\section{Heidrun Mollenkopf Jürgen Hampel Ursula Weber}

Universität Mannheim, Projekt Technikfolgen für Haushaltsorganisation und Familienbeziehungen, Tattersallstr. 2, D-6800 Mannheim

Zusammenfassung: Die Technisierung des privaten Alltags ist in den letzten Jahren zunehmend Gegenstand sozialwissenschaftlicher Diskussionen geworden. In diesem Rahmen leistet der vorliegende Aufsatz einen empirischen Beitrag zu der Frage, inwieweit technische Artefakte in familiale Lebensformen integriert werden oder diese in Richtung technischer Handlungsrationalitäten verändern. Am Beispiel spezifischer Muster gemeinsamer Zeitverwendung in Familien im Zusammenhang mit der Anschaffung neuer Geräte der Haushaltstechnik und Unterhaltungselektronik werden unterschiedliche familiale Aneignungsformen dieser technischen Geräte exemplarisch aufgezeigt.

In der techniksoziologischen Diskussion wird in den letzten Jahren verstärkt die Bedeutung von Technik im Alltag thematisiert. ${ }^{2}$ Unter anderem

${ }^{1}$ Bei diesem Aufsatz handelt es sich um die erweiterte Fassung eines von Heidrun Mollenkopf zusammen mit Jürgen Hampel und Ursula Weber verfaßten Vortrags, der im Oktober 1988 auf dem gemeinsamen Kongre $B$ der DGS, ÖGS und SGS in Zürich gehalten wurde. Er entstand im Rahmen des vom Bundesministerium für Forschung und Technologie geförderten und von Prof. Dr. Wolfgang Zapf geleiteten Projekts „Technikfolgen für Haushaltsorganisation und Familienbeziehungen".

Ziel des Projekts ist die Erarbeitung unterschiedlicher Muster der Technikausstattung und -nutzung verschiedener Haushaltstypen im Zusammenhang mit soziostrukturellen Faktoren und subjektiven Leitbildern.

Um den vielfältigen Aspekten des Themas gerecht zu werden, wurde das Projekt methodisch zweigleisig angelegt: Zum einen haben wir in fünfzig ausgewählten Haushalten im Raum Mannheim je vier Beobachtungen in halbjährlichem Abstand durchgeführt. Das in den teils standardisiert und teils offen geführten Interviews gewonnene Material, das wir qualitativ auswerten, liefert uns insbesondere die der Technikausstattung und -nutzung zugrundeliegenden subjektiven Komponenten, also Leitbilder und Einstellungen zu Technik und Umwelt, Haushaltsorganisation und Familienleben. Zum zweiten fand im Sommer 1988 eine bundesweite Repräsentativbefragung in 800 Mehrpersonenhaushalten zu inhaltlich kongruenten Fragestellungen statt, die relativ differenzierte Strukturanalysen erlaubt. Befragt wurden Personen aus Haushalten mit mindestens zwei Erwachsenen.

${ }^{2}$ Einen Einblick in diese Diskussion vermitteln die Beiträge von Lutz, Glatzer/Ostner, Hörning, Joerges, Rammert, Weingart und Zapf et al. in Lutz (1987a) sowie die Beiträge von Joerges, Hörning und Rammert in Joerges (1988a). wird danach gefragt, in welchem Maß technische Geräte alltägliche Lebensformen mehr oder weniger zwangsläufig prägen oder Interpretations- und Gestaltungsspielräume für die vielfältigen Probleme und Bedürfnisse von Akteuren offen lassen (Joerges 1988b; Rammert 1985 und 1988). ${ }^{3} \mathrm{Zu}$ dieser bisher vorwiegend auf theoretischer Ebene geführten Diskussion wollen wir einen empirischen Beitrag leisten, indem wir sozusagen die Lupe auf einen kleinen Ausschnitt der Alltagswelt richten und am Beispiel von zwei ausgewählten technischen Geräten exemplarisch der Frage nachgehen, inwieweit der Erwerb und die Nutzung technischer Geräte in die kommunitäre Alltagspraxis und den jeweils spezifischen Lebensstil von Familien integriert sind oder diese in Richtung technischer Handlungsrationalitäten verändern, wie aus technikdeterministischer Sicht zu erwarten wäre. Danach wird angenommen, daß im Verlauf des Modernisierungsprozesses die im staatlichen und wirtschaftlichen Bereich dominante instrumentelle Rationalität über diese Bereiche hinaus zunehmend auch auf traditionell verständigungsorientierte Handlungszusammenhänge übergreift. So spricht zum Beispiel Freyer (1960) vom „Dominantwerden technischer Kategorien in der Lebenswelt der industriellen Gesellschaft" und Ellul (1964) von einer sich verselbständigenden technologischen Rationalität und der Tendenz einer Perfektionierung von Techniken, die alle Lebensbereiche er-

${ }^{3}$ Zur Überformung der kulturellen Lebenswelt durch technisch-wissenschaftliche Zivilisation vgl. auch Rammert (1982: 18). 
fassen und nach ihrer Logik transformieren. ${ }^{4}$ Technische Geräte, die ja Produkte des ökonomischen Systems sind, werden unter dieser Perspektive als Träger einer solchen instrumentellen Rationalität betrachtet, die nicht nur bestimmte funktionale Alltagshandlungen objektivieren, sondern da jede Art von Artefakten gleichzeitig symbolische Qualitäten beinhaltet - auch eine Ausweitung auf eher sinnrationale Handlungsbezüge mit sich bringen (Biervert/Monse 1986: 17 und 1988: 106; Hörning 1985: 186-207, 1987: 310-314 und 1988: 51-94). Die Technisierung der Haushalte wäre demnach ein Teilaspekt dieses Prozesses.

Unseres Erachtens ist die Technisierung eines Haushalts jedoch kein unspezifisch verlaufender, technisch induzierter Diffusionsprozeß, sondern bestimmt durch familienspezifische Problemlagen, finanzielle Ressourcen, kreative und eigensinnige Praktiken der Akteure und familiale Entscheidungsprozesse. ${ }^{5}$

Natürlich können wir die Frage nach der Autonomie oder Technikbestimmtheit familialen Alltags nicht in ihrer Gesamtheit überprüfen. Wir haben uns daher auf die Zeitverwendung als einen zunehmend gesellschaftlich strukturierten Aspekt des Alltagslebens ${ }^{6}$ konzentriert und mit dem Mikro-

\footnotetext{
${ }^{4}$ Ähnliche Ansätze werden auch in neueren Veröffentlichungen vertreten. Vgl. z. B. Bammé et al. (1983), Biervert/Monse (1986 und 1988) sowie Joerges (1987).
}

Kritisch zu diesen Ansätzen äußern sich u. a. Hörning (1988) in seinem Artikel: Technik im Alttag und die Widersprüche des Alltäglichen, in Joerges (1988: 61) und Rammert (1985 und 1988).

Vgl. außerdem Rammert (1987: 320-325) in Lutz (1987). Zur „zielprägenden Potenz der Sachsysteme“ einerseits und ihrer „Multifunktionalität" andererseits vgl. Lenk/Ropohl (1978).

Als ein Beispiel dafür, wie die Thematik in der Haushaltswissenschaft aufgegriffen wird, sei verwiesen auf Kettschau/Methfessel (1985).

Erste Analysen unseres qualitativen Materials, die auf diesen Zusammenhang hindeuten, sind dargestellt in Kloé/Mollenkopf (1988).

Zur gleichen Thematik vergleiche auch Rammert (1985: 9 und 1988: $172 \mathrm{f}$ und 196f) sowie Hörning (1988: $51,54,56)$.

${ }^{6}$ Zur Zeitregulierung in modernen Gesellschaften vgl. Hohn (1984) und Rinderspacher (1985). Das Eindringen moderner Zeitorganisation in die Alltagswelt von Familien und ihre Bedeutung speziell für Kinder untersuchten z. B. Rabe-Kleberg/Zeiher (1984) und Zeiher (1988). wellenherd und dem Videorekorder zwei noch nicht lange auf dem Markt verfügbare Geräte ausgewählt, die von ihren technischen Möglichkeiten her in unterschiedlichen Bereichen großen Einfluß auf die Zeitverwendung von Familien nehmen können. ${ }^{7}$

Bei beiden Geräten haben wir untersucht, ob ihre Nutzung zu technikinduzierten Veränderungen geführt hat oder ob die Art ihrer Verwendung eher familienspezifischen Interessen und Problemlagen folgt. In einem ersten Schritt haben wir die Verbreitung dieser Geräte in Mehrpersonenhaushalten in Verbindung mit sozioökonomischem Status, Familientyp, Erwerbsstatus der Frau und Technikausstattung und -nutzung ermittelt sowie Leitbilder und Einstellungen zu ausgewählten Aspekten des Familienlebens wie zum Beispiel der Bedeutung von individueller und gemeinsam genutzter Zeit und zu Technik in bezug auf ihren alltäglichen Verwendungszusammenhang analysiert. In einem zweiten Schritt haben wir dann die konkrete Nutzung dieser Geräte untersucht. Dabei haben wir jeweils das durch die Projektanlage verfügbare quantitative und qualitative Material soweit wie möglich miteinander verknüpft und sind dabei zu folgenden Ergebnissen gekommen: ${ }^{8}$

Wie zu erwarten, wird die Ausstattung mit technischen Haushaltsgeräten von den finanziellen Möglichkeiten der Familien beeinflußt: Der Ausstattungsgrad eines Haushalts erhöht sich mit zunehmendem Einkommen. ${ }^{9}$ Eine umfangreiche Aus-

71984 war erst in $8 \%$ aller Haushalte ein Videorekorder und in $4 \%$ ein Mikrowellenherd vorhanden. Quelle: Globus Kartendienst R-5394, Dezember 1984.

Eventuelle, mit dem Besitz und der Nutzung dieser Geräte einhergehende Veränderungen können von den betreffenden Akteuren vermutlich eher wahrgenommen und beschrieben werden als bei Geräten, die schon seit Jahren wie selbstverständlich zur Ausstattung gehören.

${ }^{8}$ Zur empirischen Basis vgl. Anmerkung 1. Aus inhaltlichen Gründen beschränken sich die Analysen dieses Aufsatzes auf Partnerhaushalte $(\mathrm{N}=728)$. Differierende Zahlen in den einzelnen Tabellen sind auf fehlende Angaben oder - vor allem beim Einkommen - auf Antwortverweigerung zurückzuführen.

9 Zum Zusammenhang von Haushaltsausstattung und Einkommenssituation, Vorhandensein von Kindern und Frauenerwerbstätigkeit vgl. auch Zapf, Katrin (1986). 
Schaubild_

Ausstattung mit Haushaltstechnik nach Haushaltseinkommen

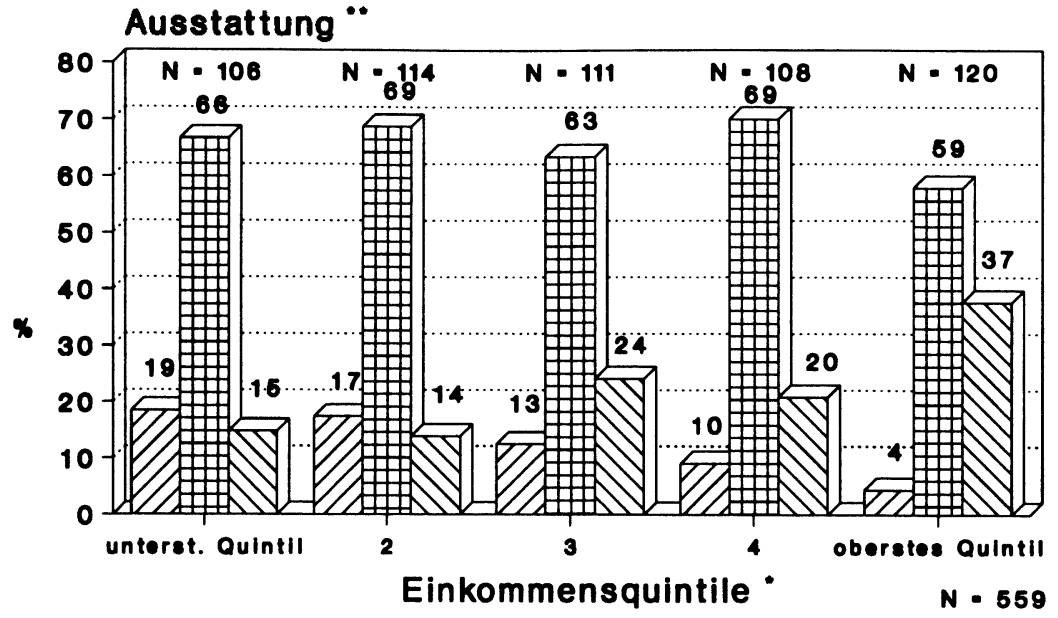

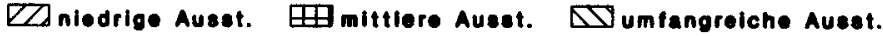

- Bedarfegewichtetes Haushalteoinkommen (vgl. Anm.)

- Der Ausetattungaindex berechnet eich nach dem Vorhandeneoin der folgenden Gerate: Mikrowellenherd, Geechirrepülmaschine, Waschmaechine Gefrlertruhe/Gefrierschrank und Whechetrockner nledrige Ausetattung: maximal oin Gerat mittlere Ausetattung: 2-3 dieser Geră te umfangreiche Ausetattung: 4-6 dieser Gerate (Vgl. Anm.)

Datenbaele: Technlkfolgensurvey 1988

Schaubild 2 Ausstattung mit Haushaltstechnik nach Familientyp

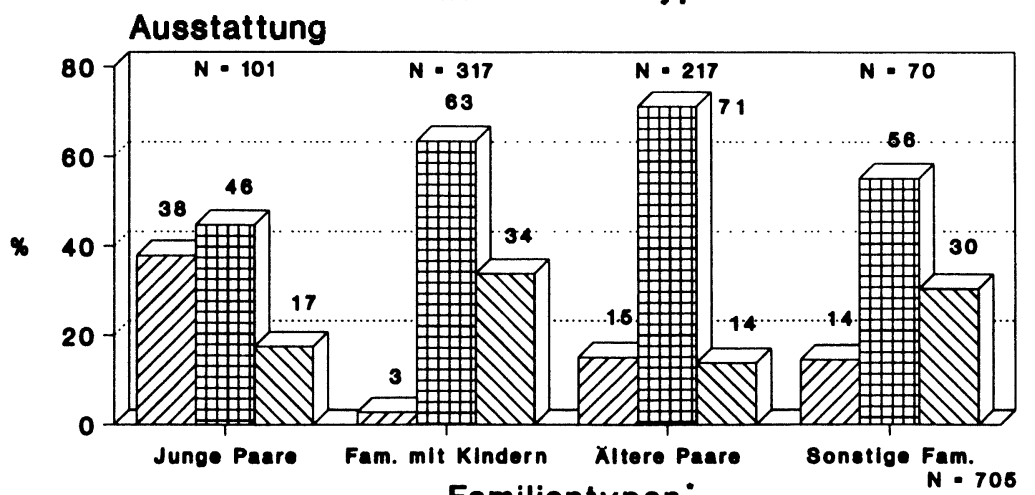

Familientypen ${ }^{\circ}$

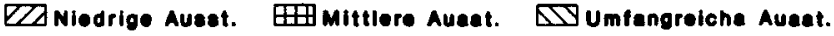

- Junge Pare: Peare, Frau unter $40 \mathrm{~J}$.

Fam. mit Kindern: Kerniamilien, mind. ein Kind unter $22 \mathrm{~J}$.

Xltere Paere: Paere, Frau uber $40 \mathrm{~J}$.

Bonstige Fam.: Familien mit nur orwechsenen Kindern oder erwelterte Familien 


\section{Schaublld 3}

\section{Ausstattung mit Haushaltstechnik nach Erwerbsstatus der Frau}

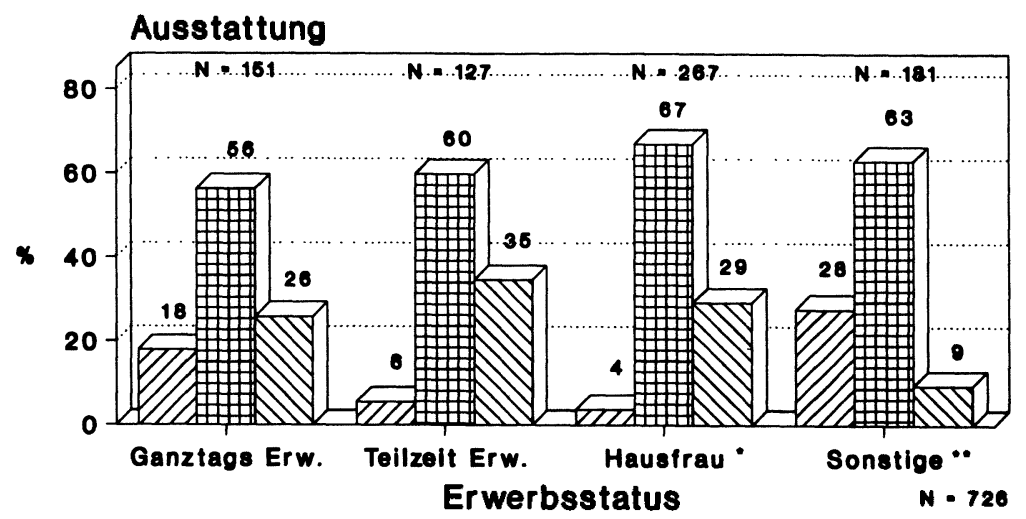

ZZ Niedrige Auset. Mittlere Auset.

- unter $60 \mathrm{~J}$.

- Rentnerinnen, Hauefrauen uber $90 \mathrm{~J}$. und

sonstlge Nichterwerbotilige

Datenbaele: Technikfolgeneurvey 1988

stattung findet sich aber immerhin auch in 15\% der Haushalte der untersten Einkommensgruppe. ${ }^{10},{ }^{11}$

Ein zumindest genauso deutlicher Zusammenhang wie mit dem Einkommen läßt sich hinsichtlich der Familienstruktur nachweisen. Wesentliches Kriterium ist hierbei, ob Kinder im Haushalt leben. Familien mit Kindern verfügen zu einem Drittel über eine umfangreiche Haushaltsausstattung, während nur jedes sechste kinderlose junge Paar

${ }^{10}$ Um einen von der Haushaltsstruktur unabhängigen Einkommenswert zu erhalten, wurde ein „bedarfsgewichtetes" Haushaltseinkommen berechnet. Die Einkommensposition eines Haushalts errechnet sich dabei als Quotient aus dem Haushaltsnettoeinkommen und einem „bedarfsorientierten Haushaltsgewicht", das auf der Grundlage der altersspezifischen Regelsätze des Bundessozialhilfegesetzes ermittelt wird (vgl. Zapf et al. 1987a: 59). Zur übersichtlicheren Darstellung wurden die Haushalte in Quintile - fünf gleich große Anteilsgruppen - zusammengefaßt.

Die Haushalte im untersten Quintil haben ein bedarfsgewichtetes monatliches Pro-Kopf-Einkommen von bis zu 879,65 DM zur Verfügung; diejenigen im zweiten Quintil bis zu 1.120,70 DM, im dritten Quintil bis zu 1.458,35 DM, im vierten Quintil bis zu 1.888,90 DM und im obersten über 1.888,90 DM. und nur jedes siebte ältere Ehepaar ohne Kinder eine solche Ausstattung besitzt.

Im Zusammenhang mit unserer Fragestellung war auch von Interesse, welche Rolle der Erwerbsstatus von Frauen spielt, weil berufstätige Frauen vermutlich über ein eingeschränkteres Zeitbudget verfügen als nicht berufstätige.

Die Ergebnisse sind hier nicht ganz eindeutig: Haushalte vollerwerbstätiger Frauen und Haus-

${ }^{11}$ Das erste methodische Problem, vor dem wir standen, war eine Operationalisierung der Technikausstattung, die es uns erlaubt, Haushalte nach diesem Merkmal in eine Rangreihe $\mathrm{zu}$ bringen. $\mathrm{Zu}$ diesem Zweck haben wir die Befragungshaushalte nach dem Besitz oder Nicht-Besitz nichttrivialer Haushaltsgeräte wie Mikrowellenherd, Geschirrspülmaschine, Waschmaschine, Gefriertruhe/Gefrierschrank und Wäschetrockner eingestuft, wobei wir für jeden Haushalt einen additiven Score bildeten. Um zu überprüfen, ob die so erhaltene Skala die Haushalte wirklich in eine Rangreihe bringt, haben wir sie einer Skalenanalyse unterzogen. Mit einem Reproduzierbarkeitskoeffizienten von 0.92 und einem Skalierbarkeitskoeffizienten von 0.62 erfüllt sie die methodischen Anforderungen, die man an eine Guttman-Skala stellt. 
frauenhaushalte haben eine geringere Ausstattung als Haushalte, in denen die Frauen einer Teilzeitbeschäftigung nachgehen. Eine Erklärung hierfür ist vermutlich, daß es sich bei letzteren wie bei den Hausfrauenhaushalten überwiegend um Familien mit Kindern handelt, während nur jede dritte ganztags arbeitende verheiratete Frau Kinder hat.

Auch die subjektive Konzeption, die man vom Haushalt und dessen Technisierung hat, scheint das Ausmaß der Ausstattung zu beeinflussen. Von den Befürwortern einer ausgiebigen Technisierung des Haushalts gehören knapp 50\% zur höchsten Ausstattungsgruppe, von denjenigen, die der Technik bei der Haushaltsführung eher eine geringere Bedeutung zumessen, dagegen nur rund $12 \%$.

Unterschiede finden sich nicht nur bei der Ausstattung mit technischen Geräten, sondern auch hinsichtlich ihrer Nutzung. So werden Geräte wie eine elektrische Nähmaschine beispielsweise relativ selten genutzt (nur zu 20\% mindestens einmal wöchentlich), eine Getreidemühle oder eine Küchenmaschine dagegen relativ häufig (Getreidemühle zu $68 \%$, Küchenmaschine zu $74 \%$ mindestens einmal wöchentlich).
Die meisten Befragten (80\%) finden - ob ihr Haushalt nun über eine geringe oder umfangreiche Ausstattung verfügt, - da $\beta$ sie gerade so viele technische Haushaltsgeräte besitzen, wie sie brauchen. Allerdings meinen von den gering ausgestatteten etwa $17 \%$, daß sie zuwenig Geräte haben, während bei den gut ausgestatteten ein etwa gleichgroßer Teil (18\%) sagt, da $\beta$ in ihrem Haushalt mehr Geräte als notwendig vorhanden sind.

Wodurch zeichnen sich nun Familien aus, die über einen Mikrowellenherd verfügen? Gelten hier die gleichen Muster wie bei der allgemeinen Haushaltsausstattung?

Nach unseren Daten besaßen 1988 rund 17\% der Mehrpersonenhaushalte in der Bundesrepublik einen Mikrowellenherd. Erwartungsgemä $\beta$ sind es vor allem Haushalte der oberen Einkommensgruppen, die im Haushaltsbereich insgesamt über eine umfangreiche Technikausstattung verfügen. $\mathrm{Ob}$ wohl sein Preis für eine weitere Verbreitung kein Hindernis mehr sein müßte (der wesentlich teurere Videorekorder hat sich schneller durchgesetzt), scheint es sich um ein Gerät zu handeln, das eher zur Abrundung einer sowieso schon guten Technikausstattung dient.

\section{Schaublld 4}

\section{Ausstattung mit Mikrowellen- herd nach Haushaltseinkommen}

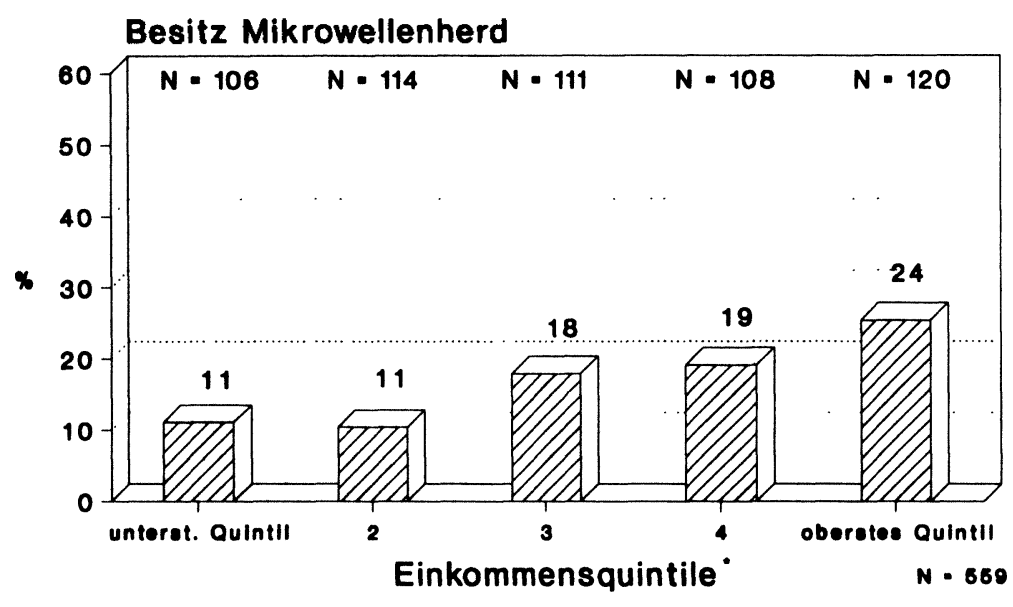

- Bedarfogowlohtotes Haushaiteoinkommen 


\section{Schaubild 5}

\section{Ausstattung mit Mikrowellenherd nach Haushaltsausstattung}

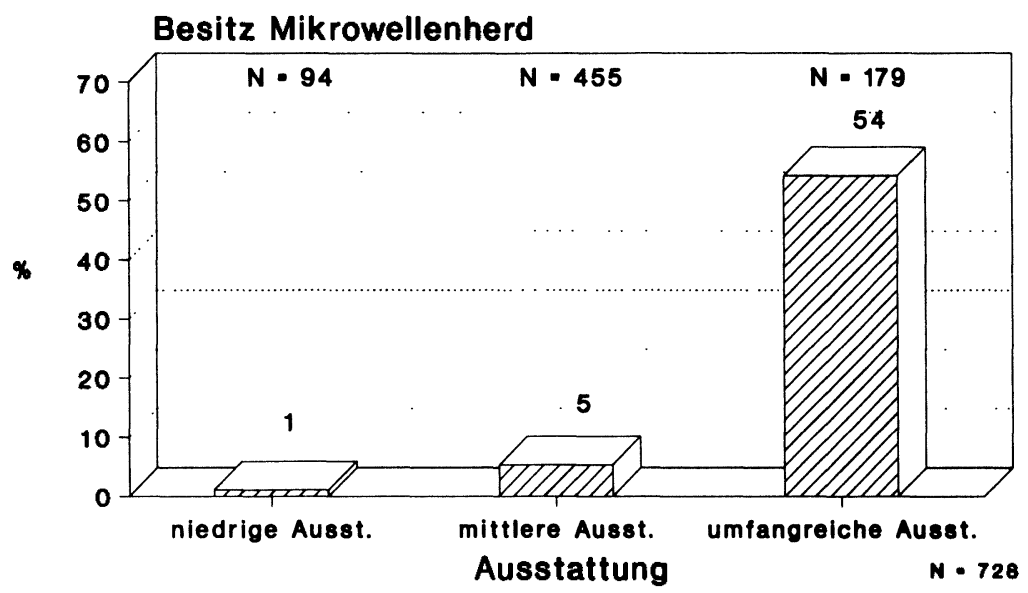

Datenbasie: Technikfolgeneurvey 1988

Schaubild 6

\section{Ausstattung mit Mikrowellenherd nach Familientyp}

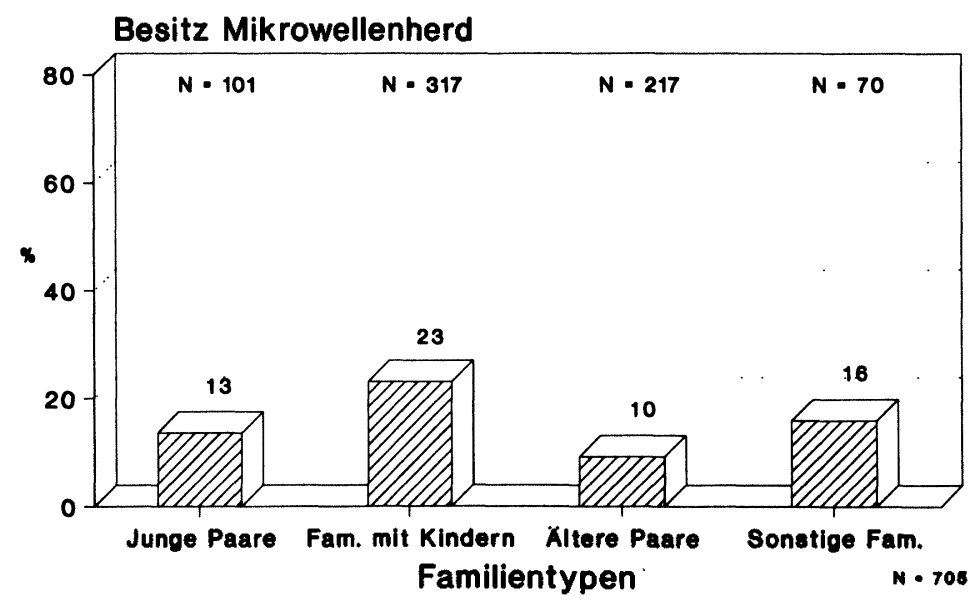

Einen deutlich wahrnehmbaren Einfluß auf den Erwerb dieses Geräts hat auch die Haushalts- und Familienstruktur. Nur in jedem neunten kinderlosen Haushalt, aber in jedem vierten Haushalt mit Kindern ist ein Mikrowellenherd vorhanden.
Hinsichtlich des Erwerbsstatus der Frau zeigt sich ebenfalls das gleiche Muster wie bei der Haushaltsausstattung als Ganzem. Wieder sind es die teilzeiterwerbstätigen Frauen, die besonders gut ausgestattet sind: fast ein Viertel von ihnen besitzt einen Mikrowellenherd. 
Eine Kombination der Variablen Familientyp und berufliche Stellung der Frau ergibt, daß es die erwerbstätigen Mütter und hier vor allem die vollzeiterwerbstätigen Mütter sind, in deren Haushalt sich überdurchschnittlich häufig ein Mikrowellenherd befindet. In Haushalten kinderloser älterer Ehepaare, in denen die Frau Hausfrau ist, haben solche Geräte dagegen eher Raritätencharakter.

Bezüglich der Einstellung zur Technisierung des Haushalts zeigt sich, daß etwa jeder dritte Haushalt, in dem eine starke Technisierung der Haushaltsführung befürwortet wird, aber nur knapp jeder zehnte technikskeptische Haushalt über einen Mikrowellenherd verfügt.

Der am häufigsten angegebene Grund für die Anschaffung eines Mikrowellenherdes ist die zeitliche Flexibilität, die das Gerät bietet. In unserer Repräsentativbefragung nannten rund 40\% die Möglichkeit einer beschleunigten Essenszubereitung. Ein starkes Drittel gab außerdem an, daß das Essen zu unterschiedlichen Zeiten Grund der Anschaffung war.

Von unseren intensiv befragten Familien wurden durchgängig die unterschiedlichen Zeiten, zu denen die einzelnen Familienmitglieder zum Essen nach Hause kommen, als ausschlaggebend für die Anschaffung des Mikrowellenherdes genannt. Als eine Folge zeigte sich in beiden Untersuchungen, $\mathrm{da} \beta$ häufiger vorgekocht wird und da $\beta$ die einzelnen Familienmitglieder sich ihre Mahlzeiten öfter selbst warm machen.

„Es kann ja nix anbrennen!“ erzählt eine Frau. „Net daß man sagt: Die passen net auf, die Männer, die laufen weg und dann brennt's an! So stellen sie's rein, stellen die Zeit ein ..." - und sie selbst wird dadurch etwas freier in der Einteilung ihrer Zeit.

(Fam. 2: Rentnerin (60), bis zur Pensionierung kaufmännische Angestellte. Ihr Mann, Frührentner (61), war technischer Angestellter. Der 20jährige Sohn steht kurz vor dem Abitur.)

Verschiedene Äußerungen deuten darauf hin, daß es nach wie vor die Frauen sind, die das Essen für die ganze Familie zubereiten und sich für das Wohlergehen der einzelnen Familienmitglieder verantwortlich fühlen. Eine Frau zum Beispiel richtet sogar für jeden in der Familie einen eigenen Teller vor:

„... da brauchen sie's nur noch reinschieben. (...) Meine Tochter ißt net so viel, er (der Mann) ißt keine Bratwurst ... Ich hab da so Kärtchen, da steht der Name drauf, und das leg ich dann oben drauf."

\section{Schaublld 7}

\section{Ausstattung mit Mikrowellenherd nach Erwerbsstatus der Frau und Vorhandensein von Kindern}

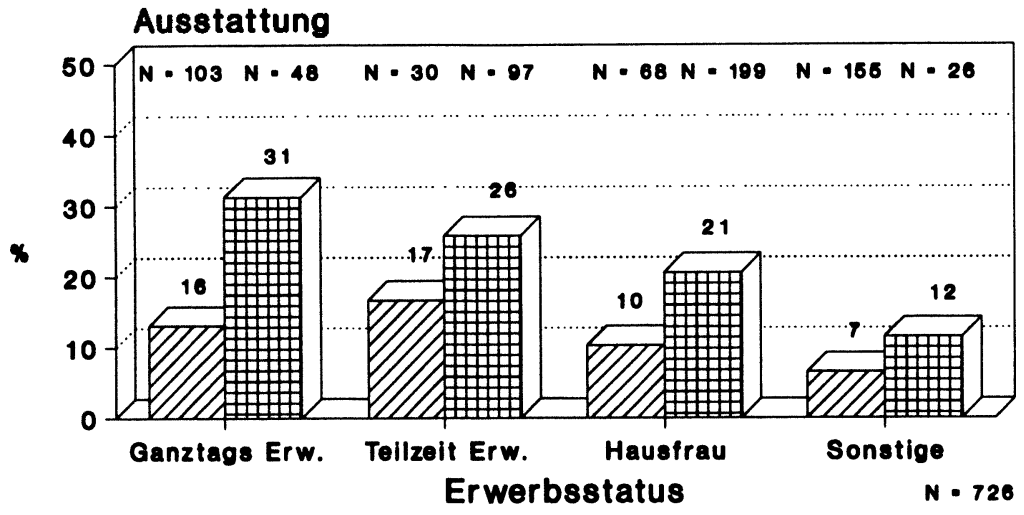

WD Ohne Kinder * Mit Kindern *

- Koln KInd unter $22 \mathrm{~J}$. Im Heuehalt

-" MIndestene eln Kind unter $22 \mathrm{~J}$. In Maushalt 
(Fam, 7: Teilzeiterwerbstätige Arbeiterin (45). Ihr Mann (46) ist Schlosser, die Kinder sind 18 und 19 Jahre alt und noch in Ausbildung).

Die traditionelle Zuständigkeit der Frauen für das Zubereiten der Mahlzeiten scheint bestehenzubleiben, aber innerhalb dieses Rahmens können sie sich durch den Mikrowellenherd möglicherweise einen etwas größeren persönlichen Spielraum schaffen.

Auch bezüglich der Kochgewohnheiten ist keine große Veränderung festzustellen. In Haushalten mit Mikrowellenherd werden lediglich nicht ganz so oft frische Lebensmittel verwendet $(64 \%)$ und die Mahlzeiten während der Woche etwas seltener selbst zubereitet $(75 \%)$ als in Haushalten ohne ein solches Gerät (70\% frische Lebensmittel, $78 \%$ selbst zubereitete Mahlzeiten). Am Wochenende gleicht dieser ohnehin schon geringfügige Unterschied sich ganz aus.

Im Hinblick auf eventuelle Veränderungen des $F a$ milienlebens durch das Vorhandensein eines Mikrowellenherdes wäre eine aus technikdeterministischer Sicht mögliche Folgerung die Annahme, daß die Haushaltsmitglieder ihre Mahlzeiten aufgrund der spezifischen Möglichkeiten des Geräts unabhängig voneinander aufbereiten und einnehmen und daß infolgedessen weniger gemeinsame Mahlzeiten stattfinden.

Von den Mikrowellenherdbesitzern der Repräsentativbefragung wurde dies fast einhellig abgelehnt. Auch von den Befragten unserer Intensivstudie berichtete keine einzige über derartige Auswirkungen. Sie hatten übereinstimmend geäußert, daß wenigstens eine gemeinsame Mahlzheit am Tag ihnen sehr wichtig ist, unter anderem, um über verschiedene Dinge zu sprechen.

„Beim Essen weniger, aber nach dem Essen sitzt man noch zusammen, wenn's die Zeit erlaubt, und da wird dann geredet. (...) Was den einen bedrückt oder erfreut hat. Oder was es so gegeben hat, was man erlebt hat. Wann sollte man sonst darüber sprechen?"

(Fam. 5: Hausfrau (50), früher Arzthelferin. Ihr Mann (58) ist technischer Angestellter, der Sohn (22) geht noch zur Schule.)

Das Gerät dient überwiegend der Anpassung an ungünstige, den Familien durch festgelegte Arbeits- und Schulzeiten vorgegebene Umstände und um in diesen Fällen, wie bereits erwähnt, die Frauen zu entlasten und den einzelnen Familienmitgliedern ein selbständiges, zeitsparendes und schonendes Aufwärmen des Essens zu ermöglichen.
„Ich sehe nicht ein, daß ich mittags von halb eins bis nachmittags um 5 in der Küche hocke, nur weil jeder anders kommt," erklärt eine Frau ihre Situation. „Da wird einmal gekocht und dann kommt der Teller in die Mikrowelle rein und wenn dann derjenige nach Hause kommt, innerhalb von einer Minute ist das Essen heiß und meine Küche ist trotzdem aufgeräumt".

(Familie 11: Teilzeiterwerbstätige Floristin (38). Ihr Mann (43) ist kaufmännischer Angestellter. Die drei Söhne $(17,14,10)$ sind Schüler.)

Getrennte Mahlzeiten scheinen also eher Ursache der Anschaffung eines Mikrowellenherdes als Folge seines Vorhandenseins zu sein. Wo gemeinsame Mahlzeiten möglich und erwünscht sind, werden sie auch mit einem solchen Gerät beibehalten.

Technik wird im Falle des Mikrowellenherdes also offensichtlich genutzt, um sich innerhalb gesellschaftlich vorgegebener Zeitstrukturen mehr Freiraum sowohl in der individuellen wie familialen Zeitverwendung zu schaffen, das heißt, um unabhängiger von extern bedingten Zeitrestriktionen zu werden.

Einen Videorekorder besitzen knapp $40 \%$ der Haushalte unserer Umfrage. ${ }^{12}$ Die Verteilungsunterschiede hinsichtlich soziodemographischer Merkmale fallen bei diesem Gerät wesentlich geringer aus als beim Mikrowellenherd. Es zeigt sich zwar, daß sowohl Haushalte mit einem Videogerät wie Haushalte mit Mikrowellenherd im Durchschnitt über eine bessere Technikausstattung verfügen als andere. Andererseits verfügt aber auch jeder vierte Haushalt mit einer ansonsten geringen Technikausstattung über einen Videorekorder, während nur jeder hundertste dieser Haushalte einen Mikrowellenherd besitzt.

${ }^{12}$ Die Geschwindigkeit, mit der sich der Videorekorder verbreitet hat, ist mit kaum einem anderen Gerät vergleichbar: Innerhalb des Unterhaltungselektronikbereichs stieg sein Anteil von 1980 bis 1982 von 8,8 auf 21,2\%. (Zum Vergleich: Der Anteil der Farbfernsehgeräte sank im gleichen Zeitraum von 39,4 auf $34,6 \%$. Quelle: Verband der Unterhaltungselektronik, FAZ Nr. 261 vom 9. 11. 1983.)

Von Arbeitnehmerhaushalten mit mittlerem Einkommen besaßen 1982 6\% einen Videorekorder, fünf Jahre später - 1987 - waren es bereits $32 \%$ (Quelle: Globus-Kartendienst R-4749 und G-7263). Bei der Geschirrspülmaschine z. B. dauerte der gleiche DiffusionsprozeB rund zehn Jahre: Ihr Anteil betrug in Arbeitnehmerhaushalten mit mittlerem Einkommen 1974 5\%, 1984 34\% und 1987 erst 47\% (Quelle: Globus-Kartendienst R-5705, R-5394 und G-7263). 
Schaubild B

\section{Ausstattung mit Videorekorder nach Haushaltsausstattung}

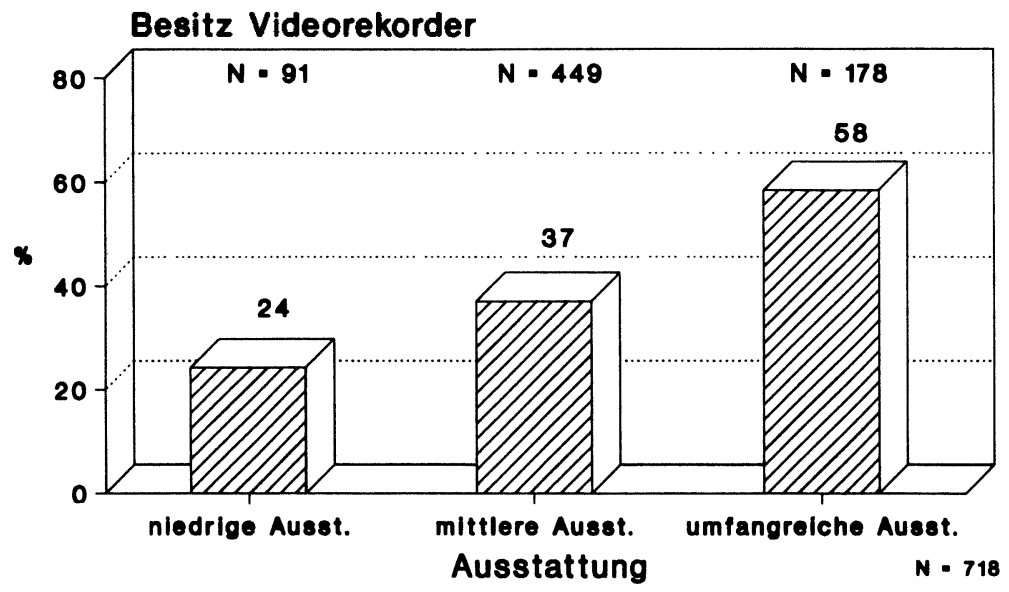

Datenbasie: Technikfolgeneurvey 1988

\section{Schaublld $\theta$}

\section{Ausstattung mit Videorekorder nach Haushaltseinkommen}

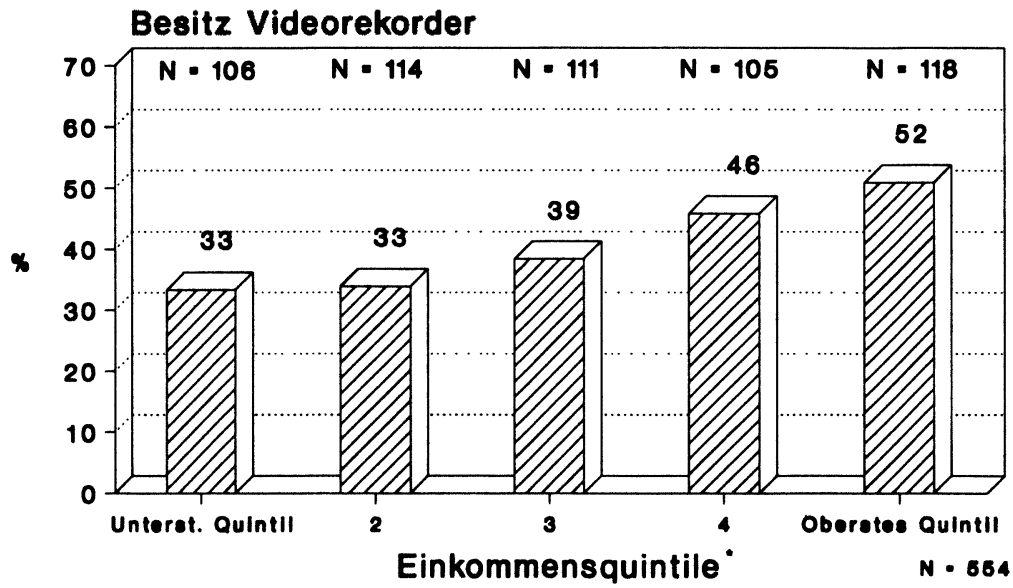

- Bedarfegowlohtetee Heushalteeinkommen

Datenbesls: Tochnikfolgensurvey 1088

Auch in bezug auf Einkommensposition, berufliche Stellung und Familientyp lassen sich die Videobesitzer keiner bestimmten Gruppe zuordnen. Es scheint jedoch einen deutlichen Alterseffekt zu geben: Von den älteren Ehepaaren besitzt nur jedes sechste einen Videorekorder.
Nach unserem qualitativen Material sind es beim Videorekorder überwiegend die Männer oder die Söhne, die an der Anschaffung interessiert waren und das Gerät nun auch am stärksten nutzen.

Die von unseren intensiv befragten Personen am häufigsten genannten Gründe für den Kauf eines 
Schaubild 10

\section{Ausstattung mit Videorekorder nach Familientyp}

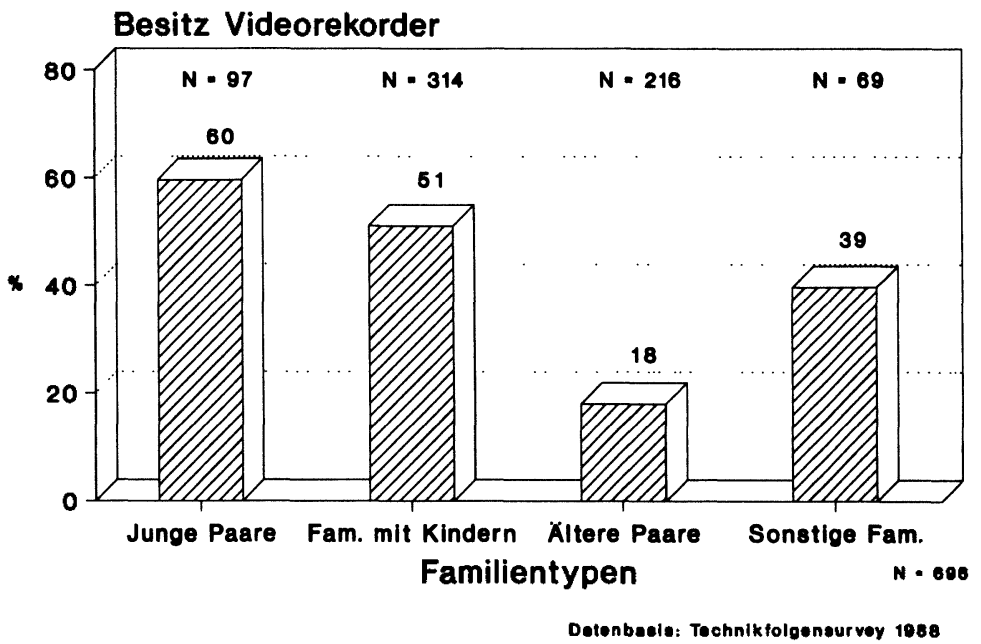

Videorekorders - und das zieht sich durch alle Familientypen und Einkommensgruppen - sind zum einen die Möglichkeit des zeitversetzten und sendezeitunabhängigen Fernsehens - sei es, weil Filme zu spät am Abend gesendet werden, weil man während der Sendezeit etwas anderes vorhat oder weil man während dieser Zeit berufstätig ist. Zum anderen ist es die Möglichkeit, Parallelsendungen aufzuzeichnen und damit sowohl der Entscheidung für oder gegen ein bestimmtes Programm auszuweichen, da die Alternativsendung ja zu einem späteren Zeitpunkt ,nach"geschaut werden kann, wie auch um unterschiedlichen Programmwünschen innerhalb der Familie gerecht zu werden.

„Man kann auch manchen Ärger mit vermeiden," meint eine Frau. „Der eine möchte des sehen, der andere des der Vater den Sport, die Tochter die Musik und ich den Krimi oder sonstwas - das kann man also alles jetzt vermeiden, indem man das eine halt aufzeichnet und dann nachschaut. (...) Unter der Woche ist ja so oft gar nichts, was einen interessiert, daß man es sich da dann anschauen kann.“

(Fam. 47: Hausfrau (51). Der Mann (65) ist Dipl--Ing. und gerade pensioniert. Die Tochter (17) ist Schülerin.)

„Mein Mann, der nimmt gern Science-Fiction-Zeug manchmal auf, und das interessiert mich nicht. Aber dann guckt er's öfters mal an, wenn ich sowieso was anderes mach'. Oder manchmal, wenn man net da ist und es interessiert einen was, dann guckt man's halt später an."
(Fam. 37: Hausfrau (40), früher Sekretärin. Ihr Mann (43) ist höherer Beamter. Drei Kinder $(12,10,5)$.)

Betrachtet man die Art der Videonutzung insgesamt, so zeigt sich, daß das Gerät am häufigsten zum Aufzeichnen und Abspielen von Fernsehsendungen verwendet wird. Die Aufzeichnungen werden in der Regel auch wieder überspielt. Lediglich ein Drittel der Befragten des Surveys gibt an, daß in ihrem Haushalt regelmäßig bespielte VideoKassetten entliehen werden. ${ }^{13}$

Die Möglichkeiten der Aufzeichnung von Bildungs- und Informationssendungen sind spezifische Interessen, die sich bei Familien der höheren Bildungsgruppe finden. Für Personen mit niedri-

${ }^{13}$ Die niedrige Zahl der Videorekorderbesitzer, die Kassetten aus kommerziellen Videotheken entleihen, verblüfft auf den ersten Blick und scheint der allgemeinen Alltagserfahrung zu widersprechen. Die Tatsache, daß in unserem Survey nach der regelmäßigen Ausleihe von Videokassetten gefragt wurde, relativiert jedoch den ersten Eindruck.

Zudem wissen wir aus der Erfahrung mit den Befragten unserer Intensivstudie, daß viele Videorekorderbesitzer die Ausleihe aus unterschiedlichen Gründen verschweigen und/oder nur unregelmäßig Kassetten aus Videotheken beziehen. Außerdem zeigte sich in diesen Interviews, daß sich die Entleihgewohnheiten der einzelnen Familienmitglieder stark unterscheiden können. 
gem Bildungsniveau scheint eher die Möglichkeit, zuhause ein nahezu unbegrenztes Unterhaltungsangebot zur Verfügung zu haben, wesentlich zu sein.

Wie im Falle des Mikrowellenherdes haben wir auch beim Videorekorder als Indikator für fremdbestimmte oder eigensinnige Techniknutzung die mit dem Vorhandensein des Geräts einhergehenden Veränderungen des Familienlebens im Hinblick auf einen autonomen oder technikdeterminierten Umgang mit Zeit untersucht. Ein so eindeutiges Kriterium wie die Beibehaltung oder Aufgabe gemeinsamer Mahlzeiten ließ sich erwartungsgemäß beim Videorekorder aufgrund seiner andersgearteten Nutzungsmöglichkeiten nicht festmachen: Beide Geräte ermöglichen eine Umorganisation von Zeit, der Videorekorder als nicht-utilitaristisches Gerät ermöglicht darüberhinaus, Zeit zu füllen. Ein wesentlicher Indikator wäre demnach die Ausweitung des Fernseh- und Videofilmkonsums.

Befragt man Haushalte mit Videorekordern nach den Veränderungen, die dieses Gerät für das $\mathrm{Fa}$ milienleben gebracht hat, werden überwiegend positive Antworten gegeben: Gezielterer Fernsehkonsum, häufigeres Abschalten bei uninteressanten Aufzeichnungen, kritischerer Umgang mit dem Fernsehprogrammangebot, eher gemeinsames Fernsehen (Einzelinteressen werden aufgezeichnet und später gezielt angeschaut - siehe die obigen Zitate) und das Zuendeführen von angefangenen Tätigkeiten und Gesprächen sind die am häufigsten genannten positiven Veränderungen durch einen Videorekorder.

Nach dem bisherigen Stand unserer Analysen lassen sich zwei unterschiedliche Arten der Videonutzung unterscheiden:

1. In Familien, in denen das Fernsehen im Familienleben und gegenüber anderen Freizeitinteressen eine eher untergeordnete Rolle spielt, scheint sich durch das Vorhandensein eines Videorekorders nichts grundsätzliches an diesen Verhaltensweisen zu ändern. Das Gerät erleichtert vielmehr eine Einpassung des vorgegebenen Programmangebots in das Aktivitäts- und Kommunikationsmuster dieser Familien.

Eine Frau äußert sich dazu folgendermaßen:

„Wir haben den Videorekorder gekauft, weil wir viel weg sind ... Wir haben einen Wohnwagen und da fahren wir freitags weg und kommen sonntags zurück ... Und da kommen dann oft Filme, die uns interessieren, und die können wir ja dann nicht sehen, weil wir grundsätzlich ohne Fernseher im Wohnwagen sind. Wir sind nicht abhängig vom Fernsehen. Das läuft nur, wenn man was sehen will. Und aus dem Grund haben wir uns den Videorekorder gekauft, daß wir die Filme aufzeichnen können und sie dann bei Gelegenheit, wenn wir Lust haben, anschauen."

(Fam. 34: Selbständige techn. Zeichnerin (42); Mann (47) techn. Angestellter, ein Sohn (17, Schüler).)

Ein Beispiel für die Nutzung des Geräts im Kontext innerfamilialer Kommunikation erzählt eine andere Frau:

„Uns ist es schon oft so gegangen, daß wir uns abends unterhalten haben ... Wenn dann was anfing, haben wir das Ding (den Videorekorder) angestellt und haben's dann später geguckt, was wir früher nicht gemacht hätten. Wir hätten dann einfach weil's uns interessiert, das Gespräch abgebrochen und hätten dann gesagt, daß wir uns hinterher weiter unterhalten könnten, was wir dann doch nicht gemacht haben, weil dann irgendwo der Faden weg ist ..."

(Fam. 51: Krankengymnastin (33), teilzeiterwerbstätig; Mann (36) techn. Angestellter, zwei Kinder $(6,1)$.)

Diese Art der Fernseh- und Videonutzung scheint schwerpunktmäßig in Familien mit mittlerem und hohem Bildungsniveau und überdurchschnittlichem Einkommen stattzufinden. Befragte mit höherer formaler Bildung gaben in der Repräsentativumfrage fast doppelt so häufig $(27 \%)$ wie Befragte mit Hauptschulabschluß (14\%) an, daß sie durch ein Videogerät mehr Zeit für die Familie haben. Unserer Meinung nach sprechen diese Befunde zur Videonutzung gegen die Annahme einer Prägung familialer Lebensformen durch technische Artefakte. Der kompetente und autonome Umgang des beschriebenen Nutzertyps mit dem Videorekorder führt im Gegenteil zu einer größeren zeitlichen und räumlichen Flexibilität.

2. Die zweite Nutzungsart haben wir vor allem in Familien beobachtet, in denen das Fernsehen bereits vor Erwerb eines Videogeräts eine größere Rolle gespielt hat. Hier findet - zum Teil auf Kosten anderer Kommunikations- und Freizeitmöglichkeiten - eher eine Ausweitung der Nutzung audiovisueller Medien sowohl durch eigenes Aufzeichnen von Sendungen wie durch das Ausleihen von Kassetten statt.

In einer von uns befragten Arbeiterfamilie läuft der Videorekorder praktisch täglich, und jeder der fünfköpfigen Familie benutzt das Gerät. Ausgeliehen werden nach Aussage der Frau vor allem Actionfilme.

„Wieviel in der Woche? Moment ... zweimal in der Woche fahre ich rein (in die Stadt), dann kann man jedesmal drei Kassetten mitnehmen. Sechs Kassetten kann man sagen in der Woche." 


\section{Schaublld $11{ }^{14}$ \\ Videonutzung in Familien mit Kindern nach Bildungsniveau}

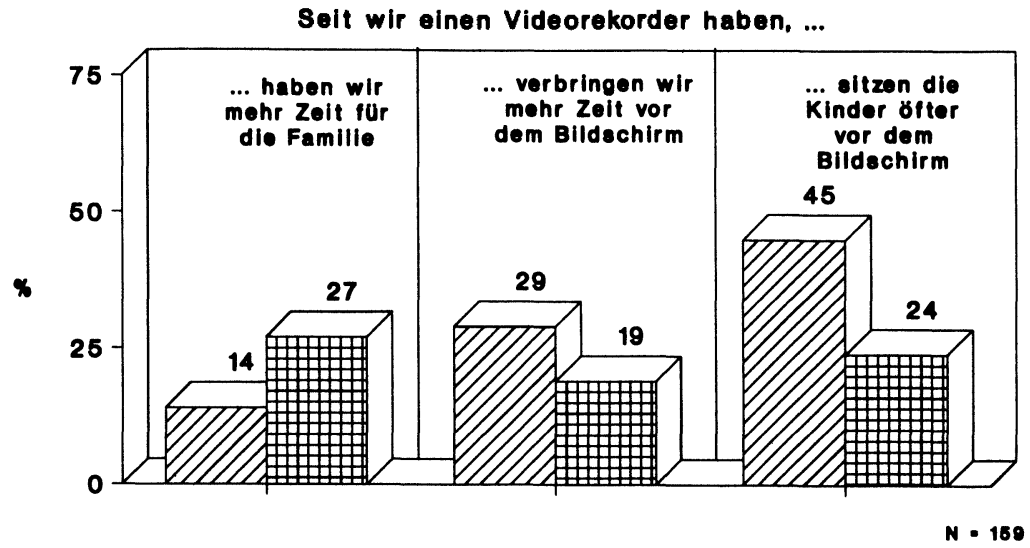

EZ7 Hauptschulabschl. Fachhochsch.,Abitur

Datenbasls: Technikfolgeneurvey 1988

(Fam. 1: Familie mit drei Kindern (15, 7 und 5), Mann Arbeiter (35), Frau (35) gelernte Näherin, arbeitet stundenweise als Putzfrau.)

Ein anderer Befragter berichtet, daß vor allem bei seinem Sohn der Fernsehkonsum gestiegen ist:

„Es wird mehr geguckt. An den Samstagen, wo sie (der Sohn und seine Freunde) vorgehabt haben ins Kino zu gehen - sie gehen als auch mal noch ins Kino - holen sie sich vielleicht einen Film heim und gucken, wo sie net geguckt hätten."

Und besonders Spaß macht dem Sohn nach Aussage des Vaters, , daß er halt gucken kann, die Filme da - schön im Sessel hocken mit Fernbedienung - braucht net hinlaufen, braucht bloß Kassette einlegen - kann spulen, Bildstopp, Vor-, Rücklauf, alles fernbedienen - bloß beim Auswurf muß man noch aufstehen."

(Fam. 7: Familie mit zwei Kindern (Tochter 19, Sohn 18, in Ausbildung), Mann Schlosser (46), Frau (45) Arbeiterin, teilzeiterwerbstätig.)

Diese Art der Videonutzung scheint stärker in Familien mit vergleichsweise niedrigem Bildungsniveau und geringen finanziellen Ressourcen verbreitet zu sein. Das Betrachten von Fernsehsendungen und Videofilmen dient hier teilweise zur

${ }^{14}$ Befragte mit Realschulabschluß sind in diesem Schaubild nicht berücksichtigt, da die Aussagen dieser Bildungsgruppe zu einzelnen Items im Gegensatz zu den anderen kein einheitliches Bild aufweisen.
Ablenkung von Problemen und als Ersatz für andere Beschäftigungen. So antwortet ein Rentner, dessen Frau zum Zeitpunkt des Interviews wegen einer Krebsoperation im Krankenhaus lag, auf die Frage nach den am häufigsten ausgeliehenen Videofilmen:

„Verschieden. Abenteuerfilme, daß man auf andere Gedanken kommt. Wildwest oder sowas."

Und er meint, die Ausgabe für das Gerät habe sich gelohnt:

„Ja, jetzt besonders, dadurch, daß meine Frau nicht da ist." (Fam. 43: Rentner (66), früher Monteur.)

Nach den Daten der Repräsentativbefragung steigt der audiovisuelle Medienkonsum in Familien mit niedrigem Bildungsniveau stärker als in anderen, und Befragte mit Hauptschulabschluß geben fast doppelt so häufig wie Befragte mit höherer Bildung an, daß ihre Kinder mehr Zeit vor dem Bildschirm verbringen.

In diesen Fällen, d. h. also bei Familien, in denen der Videorekorder Tendenzen zu einem vorwiegend passiven Umgang mit Zeit unterstützt und den persönlichen Spielraum lediglich in diesem Rahmen in bezug auf eine zeitliche und umfangmäßige Erweiterung des Konsumangebots an Unterhaltung vergrößert, könnte man unseres Erachtens am ehesten mit einer gewissen Begründung von technikinduzierten Veränderungen sprechen. 
Es scheint jedoch auch hier so zu sein, $\mathrm{da} ß$ nicht die Technik als solche zu Verhaltensänderungen führt, sondern daß sie bei bestimmten gegebenen Bedingungen - in diesem Fall mangelnden Kompetenzen und restringierten Ressourcen - als eine Art verstärkendes Medium vorhandene Tendenzen noch intensiviert. ${ }^{15}$

\section{Schlußbemerkungen}

Aufgrund der bisherigen Auswertung unseres Materials möchten wir unsere Ausgangsfrage folgendermaßen beantworten:

Wenn man Aussagen darüber machen will, ob technische Geräte in Familien eher autonom oder eher technikbestimmt genutzt werden und ob Technik zu einem Übergreifen instrumenteller Rationalität auch auf kommunikative Handlungsbereiche führt, muß man differenzieren, um welche Geräte es sich handelt und auf welche Voraussetzungen diese Geräte in Familien treffen.

Unsere Ergebnisse deuten darauf hin, daß Technik zumindest in Teilbereichen alltagsweltlicher Lebenszusammenhänge durchaus eigensinnig und nach familienspezifischer Rationalität angeschafft und genutzt wird, wobei diese Aneignung je nach Familie ganz unterschiedlich aussehen kann. Jedenfalls haben Familien sich Deutungen zurechtgelegt, die nicht mit Entfremdungsthesen konform gehen. Damit wollen wir natürlich nicht ausschlieBen, daß bereits im Vorfeld sowie im weiteren Verlauf eine Beeinflussung von Bewußtsein und Orientierungsmustern stattfinden kann. Die Veränderungen familialen Alltags durch eine zunehmende Technisierung scheinen jedoch subtiler zu wirken als es von den Akteuren unmittelbar erlebt und berichtet wird.

${ }^{15}$ Diese beiden Arten der Fernseh- und Videonutzung weisen in eine ähnliche Richtung wie Ergebnisse der ZDF-Medienforschung, die in einer Untersuchung „Video im Alltag“ zwei Typen von Videonutzern fand: Die „extravertierten“ Videonutzer werden durch den Videorekorder mobiler und flexibler. Sie unternehmen viel in ihrer Freizeit, auch außer Haus. Kennzeichnend sind überdurchschnittliche Bildung, höheres Einkommen, gute Geräteausstattung und eine Ehefrau, die häufig mitverdient. Die ,introvertierten“ Videonutzer verbringen wenig Zeit außer Haus und empfinden oft Langeweile. Der Videorekorder führt dazu, daß sie mehr Zeit vor dem Bildschirm verbringen. Kennzeichnend sind viele Kinder, wenig Geräte im Haushalt, Verlangen nach passiver Unterhaltung und selten eine berufstätige Frau. (ZDF-Schriftenreihe 1985).

\section{Literatur}

Bammé, A./Feuerstein, G./Genth, R./Holling, E./Kahle, R./Kempin, P., 1983: Maschinen-Menschen MenschMaschinen. Grundrisse einer sozialen Beziehung. Reinbek: Rowohlt.

Biervert, B./Monse, K., 1986: Technik und Alltag als Interferenzproblem. Forschungsgruppe Sozialökonomischer Wandel. Diskussionspapier 1/86. Wuppertal.

Biervert, B./Monse, K., 1988: Technik und Alltag als Interferenzproblem. S. 95-119 in: Joerges, B. (Hrsg.), Technik im Alltag. Frankfurt: Suhrkamp.

Ellul, J., 1954: La technique ou l'enjeu du siècle. Paris: Librairie Armand Colin.

FAZ Nr. 261 vom 9. 11. 1983.

Freyer, H., 1960: Über das Dominantwerden technischer Kategorien in der Lebenswelt. Mainz, Akademie der Wissenschaften und der Literatur, Heft 7: 539-551.

Glatzer, W./Berger-Schmitt, R. (Hrsg.), 1986: Haushaltsproduktion und Netzwerkhilfe. Frankfurt: Campus.

Glatzer, W./Ostner, I., 1987: Einführung in die Thematik. S. 199-203 in: Lutz, B. (Hrsg.), Technik und sozialer Wandel. Frankfurt/New York: Campus.

Hohn, H.-W., 1984: Die Zerstörung der Zeit. Frankfurt: Fischer

Hörning, K. H., 1985: Technik und Symbol. Ein Beitrag zur Soziologie alltäglichen Technikumgangs. Soziale Welt, Heft 2/36: 186-207.

Hörning, K. H., 1987: Technik und Alltag: Plädoyer für eine Kulturperspektive in der Techniksoziologie. S. 310-314 in: Lutz, B. (Hrsg.), Technik und sozialer Wandel. Frankfurt/New York: Campus.

Hörning, K. H., 1988: Technik im Alltag und die Widersprüche des Alltäglichen. S. 51-94 in: Joerges, B. (Hrsg.), Technik im Alltag. Frankfurt: Suhrkamp.

Joerges, B., 1987: Technik im Alltag oder: Die Rationalisierung geht weiter. S. 305-309 in: Lutz, B. (Hrsg.), Technik und sozialer Wandel. Frankfurt/New York: Campus.

Joerges, B., (Hrsg.), 1988a: Technik im Alltag. Frankfurt: Suhrkamp.

Joerges, B., 1988b: Technik im Alltag. Annäherung an ein schwieriges Thema. S. 7-19 in: Joerges, B. (Hrsg.), Technik im Alltag. Frankfurt: Suhrkamp.

Joerges, B., 1988c: Gerätetechnik und Alltagshandeln. Vorschläge zur Analyse der Technisierung alltäglicher Handlungsstrukturen. S. 20-50 in: Joerges, B. (Hrsg.), Technik im Alltag. Frankfurt: Suhrkamp.

Kettschau, I./Methfessel, B., 1985: Bedeutung und Auswirkung neuer Technologien im privaten Haushalt. Hauswirtschaft und Wissenschaft, 33. Jg., Heft 2: 85-87.

Kloé, U./Mollenkopf, H., 1988: Technik - Mittel zu jedem Zweck? Erste Ergebnisse und Interpretationsansätze aus qualitativen Familienbeobachtungen. Verbund Sozialwissenschaftliche Technikforschung, Mitteilungen 2/1988. München: 7-63.

Lenk, H./Ropohl, G. 1978: Technik im Alltag. In: KZfSS, Sonderheft 20: 265-298. 
Lutz, B. (Hrsg.), 1987a: Technik und sozialer Wandel. Verhandlungen des 23. Deutschen Soziologentages in Hamburg 1986. Frankfurt/New York: Campus.

Lutz, B. 1987b: Das Ende des Technikdeterminismus und die Folgen - soziologische Technikforschung vor neuen Aufgaben und neuen Problemen. S. 34-52 in: Lutz, B. (Hrsg.), Technik und sozialer Wandel. Verhandlungen des 23. Deutschen Soziologentages in Hamburg 1986. Frankfurt/New York: Campus.

Rabe-Kleberg, U./Zeiher, H., 1984: Kindheit und Zeit. über das Eindringen moderner Zeitorganisation in die Lebensbedingungen von Kindern. Zeitschrift für Sozialisationsforschung und Erziehungssoziologie., Heft 1/84: 29-43.

Rammert, W., 1982: Technik und Gesellschaft. S. 13-47 in: Technik und Gesellschaft. Jahrbuch 1, Frankfurt: Campus.

Rammert, W., 1985: Technisierung im Alltag. Theoriestücke für eine spezielle soziologische Perspektive. Universität Bielefeld, Forschungsschwerpunkt ,Zukunft der Arbeit“. Arbeitsbericht Nr. 12.

Rammert, W., 1987: Der nicht zu vernachlässigende Anteil des Alltagslebens selbst an seiner Technisierung. S. 320-325 in: Lutz, B. (Hrsg.), Technik und sozialer Wandel. Frankfurt/New York: Campus.
Rammert, W., 1988: Technisierung im Alltag. Theoriestücke für eine spezielle soziologische Perspektive. S. 165-197 in: Joerges, B. (Hrsg.), Technik im Alltag. Frankfurt: Suhrkamp.

Rinderspacher, J. P., 1985: Gesellschaft ohne Zeit. Frankfurt/New York: Campus.

Weingart, P., 1987: Differenzierung der Technik oder Entdifferenzierung der Kultur. Zur Bedeutung alltäglicher Verwendungszusammenhänge für die Technikentwicklung. S. 315-319 in: Lutz, B. (Hrsg.), Technik und sozialer Wandel. Frankfurt/New York: Campus.

Zapf, K., 1986: Soziale Technikfolgen in den privaten Haushalten. S. 207-241 in: Glatzer, W./BergerSchmitt, R. (Hrsg.), Haushaltsproduktion und Netzwerkhilfe. Frankfurt: Campus.

Zapf, W. et al, , 1987a: Individualisierung und Sicherheit. München: Beck.

Zapf, W./Breuer, S./Hampel, J., 1987b: Technikfolgen für Haushaltsorganisation und Familienbeziehungen. S. 220-232 in: Lutz, B. (Hrsg.), Technik und sozialer Wandel. Frankfurt/New York: Campus.

ZDF-Schriftenreihe, Heft 32, Medienforschung: Video im Alltag. Münster/München 1985.

Zeiher, H., 1988: Verselbständigte Zeit - selbständigere Kinder? Neue Sammlung, 28. Jg., Heft 1: 75-92. 